\title{
Numerical analysis of the urine flow in a stented ureter with no peristalsis
}

\author{
Hyoung-Ho Kim ${ }^{\text {a }}$, Young Ho Choi ${ }^{\text {b, }}$, Seung Bae Lee ${ }^{\mathrm{c}}$, Yasutaka Baba ${ }^{\mathrm{d}}$, Kyung-Wuk Kim ${ }^{\mathrm{a}}$ \\ and Sang-Ho Suh ${ }^{\mathrm{a}}$ \\ ${ }^{a}$ Department of Mechanical Engineering, Soongsil University, 369 Sangdo-Ro, Dongjak-gu, Seoul, \\ 156-743, Korea \\ ${ }^{b}$ Department of Radiology, Seoul National University Boramae Hospital, 425 Shindaebang-2-dong, \\ Dongjak-gu, Seoul,156-707, Korea \\ ${ }^{c}$ Department of Urology, Seoul National University Boramae Hospital, 425 Shindaebang-2-dong, \\ Dongjak-gu, Seoul,156-707, Korea \\ ${ }^{d}$ Department of Radiology, Ryokusenkai Yonemori Hospital, 1-7-1, Yojiro, Kagoshima-shi, Kagoshima, \\ 890-0062, Japan
}

\begin{abstract}
A ureteral stenosis or occlusion causes the disturbance of normal urine flow and results in renal failure. Ureteral stents are used to relieve the stagnation of urine in the upper urinary tract. Peristalsis in the ureter, which occurs to help urine flow, becomes to weaken when a stent is inserted and effective peristalsis disappears as time goes on, and a stented ureter seems to be tubular and curved in the human body. Double J stents, which are manufactured by many medical companies and are used widely these days, have different geometries of side holes in the stent shafts. In total, 12 models — six curved models of a stented ureter according to different numbers and positions of side holes and ureteral and stent stenoses and another six straight models for comparison with the curved ones - were made based on the data collected from 19 men. The flow rate and pattern in the stented ureter were evaluated using computational fluid dynamics (CFD). According to the results, curved models reflecting the human anatomy seem to be more desirable in the CFD simulation of urine flow and must be good for evaluating the effect of geometrical variations in stent design on urine flow.
\end{abstract}

Keywords: Ureter, stent, flow, CFD, numerical analysis

\section{Introduction}

Urine produced in the kidney is transported to the bladder through the ureter. Ureteral peristalsis controls urine transport from the kidney to the bladder partially, along with hydrodynamic forces [1]. A stenosis or occlusion in the ureter by benign or malignant diseases induces insufficient urine flow from the kidney to the bladder, despite peristaltic efforts, resulting in renal failure. One treatment for the disturbed urine flow is the insertion of a ureteral stent in the upper urinary tract. The insertion is

\footnotetext{
* Address for correspondence: Young Ho Choi, Department of Radiology, Seoul National University Boramae Hospital, 425 Shindaebang-2-dong, Dongjak-gu, Seoul, 156-707, Korea. Tel.: 82-2-870-2534; Fax: 82-2-870-3863; E-mail: cyho50168@naver.com.
} 
performed by an endoscopic approach of reaching the ureter through the urethra or by percutaneous nephrostomy.

There are two kinds of ureteral stents used these days: metallic and plastic stents [2-4]. Metallic stents are applied for ureteral tight stenosis or occlusion by periureteral malignancies and its applications are not so wide compared to plastic stents. Plastic stents have been made of silicone, polyethylene, and polyurethane, and some have been designed in the form of a double $\mathrm{J}$ at both ends of the stent to prevent stent migration and to anchor them in the renal pelvis and bladder.

In the stented ureter, urine flows through both the inner bore space of the stent (luminal or intraluminal flow) and the outer ureter space of the stent (extraluminal flow), and detouring flows happen around the stenosed or occluded segment in the ureter. Urine around the stent finds a detour before the segment, goes into the stent, and it comes out around the stent again after the segment. The side holes of a stent exist to help the detour, but its number and position in a stent vary and depend on the medical companies developing the stents.

Although ureteral stents are inserted to relieve the stagnation of urine flow due to the stenosis or occlusion of the ureter, indwelling of a ureteral stent itself could be a factor of insufficient urine flow [5-7] because it prevents peristalsis in the upper urinary tract, as well as results in the loss of effective ureteral peristalsis in the end. Previous studies [8-12] evaluated the urine flow in a stented ureter using the model of a straight ureter and a double $\mathrm{J}$ stent. However, the model was not based on the human ureter anatomy, especially the ureter curvature in the human body and the ureter diameter in a condition of no ureteral peristalsis. Here, it was considered that the ureter behaves as a non-uniform passively distensible tube in the absence of peristalsis [1] and data from human beings were collected. We made the model of a stented ureter and analyzed the flow rate and pattern of the model.

\section{Methods}

\subsection{Modeling of stented ureters (Figure 1)}

Two-dimensional axial computed tomography (CT) data of the upper urinary tract, including the kidney, ureter, and bladder, collected from 19 men who did not have any clinical history of urologic diseases were used for the modeling of a stented ureter, along with a double $J$ stent from Cook Medical Company as an example of a ureteral stent.

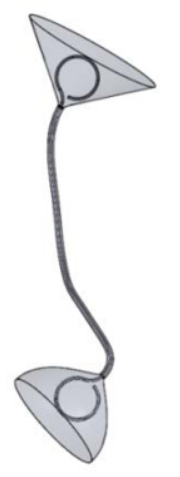

(a)

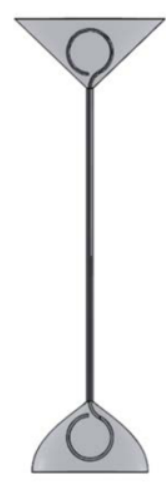

(b)

Fig. 1. Models of curved (a) and straight (b) stented ureters. 
The CT image data in the form of DICOM files were sent to a three-dimensional reconstruction program, Amira 5.6, and the upper urinary tract, extracted semi-automatically in a condition of a contrast number greater than 216, was stored in the form of STL files. In a modeling program, Ansys ICEM, the renal pelvis above the ureteropelvic junction and the bladder below the vesicoureteral junction were removed, and only the ureter was isolated.

The upper and lower ends of the ureter were connected with a straight line and the distance between them was measured on the line. The line was divided into 15 equal segments, and 16 points, including the upper and lower ends of the ureter, were achieved. The axial cross sections of the ureter at the points, perpendicular to both the plane where the men laid down for CT scanning and the plane where the scanning was headed along, were isolated. The shape of the cross sections was not typically round and therefore, the diameter of the ureter was calculated with the area measured, assuming the cross section is a circle. In addition, the axial distance between the adjoining two cross sections was measured on the straight line connecting the centers of the cross sections. A ureter model was constructed by averaging the data of the ureteral lengths and diameters from 19 cases. The diameter and length of the model were $4.57 \mathrm{~mm}$ and $226.21 \mathrm{~mm}$, respectively. The curvature of the ureter model was also established by averaging the data collected from the cases.

The renal pelvis and bladder connected to the ureter were made using the data from one of 19 cases (No. 4). However, the bladder should have been removed from the model because the limited capacity of the personal computers in the laboratory was found during the CFD simulation. The shape of the renal pelvis was a funnel, with upper and lower diameters and a height of $109 \mathrm{~mm}, 4.57 \mathrm{~mm}$, and 50 $\mathrm{mm}$, respectively.

The ureteral stent consists of two coiled ends and a shaft. The shaft has multiple side holes that provide a flow diversion from the stent to the ureter or vice versa, and the coiled ends also have multiple side holes and an end hole, which play a role in urine flow in and out of the stent in the kidney and bladder. Here, the side holes and end hole in the proximal and distal coils of the stent would be called ports, to be differentiated from the side holes in the shaft of the stent. In the study, the outer and inner diameters of the stent were $2 \mathrm{~mm}$ and $1 \mathrm{~mm}$, respectively. The shaft was $226.21 \mathrm{~mm}$, as long as the ureter, and it has 0,11 , or 22 side holes. Each coil was round with a diameter of $32 \mathrm{~mm}$ and five ports. The diameter of the side holes and ports was $1 \mathrm{~mm}$.

Four different types of stents with regard to side holes were made for the evaluation of the effect of the number and position of side holes on urine flow. The first type is a stent without any side holes. The others are stents with side holes at a $2-\mathrm{cm}$ interval and 180-degree rotation (second type), a 1-cm interval and 180-degree rotation (third type), and a 1-cm interval and 90-degree rotation (fourth type). Annular elevations in the ureter and stent (fifth and sixth types) mimicking ureteral stenoses and instent encrustations were made on the second type of stent for the evaluation of the effect of ureteral stenosis and stent encrustation on urine flow. They induced a 5\% decrease in the ureteral diameter and a $30 \%$ decrease in the inner diameter of the stent.

In modeling the six stented ureters, the stent goes along the axis of the ureter and is always located in the center of the ureter on axial cross sections. The models were not straight ureters but curved ones, because they were based on the human anatomy. Six additional models of straight stented ureters were made for comparisons with the curved ones. The 12 stented ureter models have an identical diameter and length to the ureter and stent (Table 1).

\subsection{Governing equations for fluid flow and numerical simulation}

The governing equations - the continuity equation and the Navier-Stokes equation-were converted 
Table 1

Specifications of curved and straight stented ureters

\begin{tabular}{|c|c|c|c|c|c|}
\hline Case & Ureter & $\begin{array}{l}\text { Number of side } \\
\text { holes }\end{array}$ & $\begin{array}{l}\text { Angular position of side } \\
\text { holes }\end{array}$ & $\begin{array}{l}\text { Interval of side } \\
\text { holes }\end{array}$ & Stenosis \\
\hline 1 & Curved & 0 & - & - & None \\
\hline 2 & Curved & 11 & $0^{\circ}, 180^{\circ}$ & $2 \mathrm{~cm}$ & None \\
\hline 3 & Curved & 22 & $0^{\circ}, 180^{\circ}$ & $1 \mathrm{~cm}$ & None \\
\hline 4 & Curved & 22 & $0^{\circ}, 90^{\circ}, 180^{\circ}, 270^{\circ}$ & $1 \mathrm{~cm}$ & None \\
\hline 5 & Curved & 11 & $0^{\circ}, 180^{\circ}$ & $2 \mathrm{~cm}$ & $\begin{array}{l}\text { Two in ureter (between } 4 \& 5,7 \\
\& 8 \text { side holes) }\end{array}$ \\
\hline 6 & Curved & 11 & $0^{\circ}, 180^{\circ}$ & $2 \mathrm{~cm}$ & $\begin{array}{l}\text { Two in stent (between } 4 \& 5,7 \& \\
8 \text { side holes) }\end{array}$ \\
\hline 7 & Straight & 0 & - & - & None \\
\hline 8 & Straight & 11 & $0^{\circ}, 180^{\circ}$ & $2 \mathrm{~cm}$ & None \\
\hline 9 & Straight & 22 & $0^{\circ}, 180^{\circ}$ & $1 \mathrm{~cm}$ & None \\
\hline 10 & Straight & 22 & $0^{\circ}, 90^{\circ}, 180^{\circ}, 270^{\circ}$ & $1 \mathrm{~cm}$ & None \\
\hline 11 & Straight & 11 & $0^{\circ}, 180^{\circ}$ & $2 \mathrm{~cm}$ & $\begin{array}{l}\text { Two in ureter (between } 4 \& 5,7 \\
\& 8 \text { side holes) }\end{array}$ \\
\hline 12 & Straight & 11 & $0^{\circ}, 180^{\circ}$ & $2 \mathrm{~cm}$ & $\begin{array}{l}\text { Two in stent (between } 4 \& 5,7 \& \\
8 \text { side holes) }\end{array}$ \\
\hline
\end{tabular}

to algebraic equations by the discretization method using the finite volume method. To investigate the flow phenomenon in the ureter, Ansys CFX was used. The continuity and momentum equations are shown in Eqs. (1) and (2), where $\rho, \mu, \vec{u}$, and $p$ are the density, dynamic viscosity, velocity vector, and pressure, respectively. The hybrid differential scheme is adopted for the discretization of the convective terms, as well as the SIMPLEC algorithm for treating the pressure terms in the momentum equations.

$$
\begin{gathered}
\nabla \cdot \vec{u}=0 \\
\rho\left[\frac{\partial \vec{u}}{\partial t}+(\vec{u} \cdot \nabla) \vec{u}\right]=-\nabla p+\mu \nabla^{2} \vec{u}
\end{gathered}
$$

A urine viscosity and density of $0.654 \mathrm{mPa} \cdot \mathrm{s}$ and $1,003 \mathrm{~kg} / \mathrm{m}^{3}$, respectively, were applied here. The urine used in the numerical simulation is similar to water in density and dynamic viscosity because most urine consists of water. The density and dynamic viscosity of urine are $1,003-1,035 \mathrm{~kg} / \mathrm{m}^{3}$ and $0.635-0.797 \mathrm{mPa} \cdot \mathrm{s}$, respectively, at a temperature of $37^{\circ} \mathrm{C}$. The viscosity changes according to temperature, but the change is negligible in the range of a body temperature of $35-40.5^{\circ} \mathrm{C}$, and the urine could be considered a non-compressible and Newtonian fluid. The pressure was used for the boundary condition here; the inlet $(48.9 \mathrm{~Pa})$ and outlet $(0 \mathrm{~Pa})$ were specified according to the reference study [8]. It was for all the 12 models to enable the comparison between the models.

To generate meshes in the ureter models, the same size of mesh was set up and prism mesh was used for ports of stent coils and for the side holes of a stent shaft. The meshes were generated with Ansys ICEM. The greater the number of side holes, the smaller the area for meshing. Therefore, the number of nodes and elements for a model depended on the model types, and the number of nodes ranged from 1.8 million to 2.2 million, while that of elements ranged from 7.7 million to 9.5 million. 


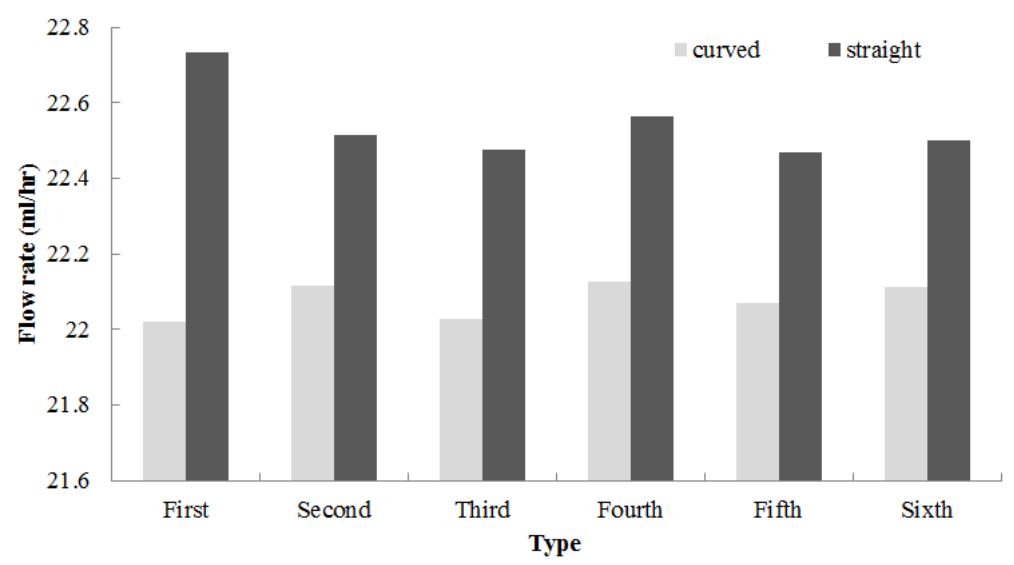

Fig. 2. Total flow rates in curved and straight stented ureters.

\section{Results and discussion}

\subsection{Total flow rate}

Figure 2 shows the total flow rate carried by the inner bore space and outer ureter space of the stent in the 12 stented ureter models. The unit of flow rate was $\mathrm{ml} / \mathrm{hr}$. The total flow rate of the straight models was a little greater than that of the curved models. The fluid flowing in a tube might have more resistance in a curved than in a straight model.

Among the straight models, the total flow rate in the first type (case 7) was just a little greater than that in the other five types (cases 8-12). However, among the curved models, the total flow rate in the first type (case 1) was just a little lesser than that in the other five models (cases 2-6). It might be inferred from the result that the side holes in a stent shaft are an obstacle to the urine flow in the straight ureter and they are a helper, enhancing the urine flow in the curved ureter. In the human body, the ureter is not straight but curved, which means side holes in a stent could play a role in increasing the urine flow through the stented ureter.

The change in the number and position of side holes in a stent shaft or the existence of ureteral or stent stenosis did not affect the total flow rate, which is demonstrated in Figure 2. However, a further study about the effect of side holes on urine flow using various changes in the geometry of side holes might demonstrate different results, and this is necessary in the future.

\subsection{Luminal and extraluminal flow rates and patterns}

The flow rate and flow pattern in the inner bore space and outer ureter space of the stent are presented in Figures 3-7. The individual flow rates - luminal, extraluminal, and total flow rates-in case 1 are shown in Figure 3. In the renal pelvis, urine flows into the inner space of the stent mainly through the fifth port, which is the last port in the coil of the stent and the nearest port to the ureter, and partially through the fourth port. Minimal urine inflow to the luminal space through the third port was demonstrated on the streamline of urine flow. Figure 4 shows the individual flow rates in case 2. There is no difference from case 1 in the pattern of urine inflow to the inner space of the stent in the renal pelvis through the ports in the proximal coil of the stent. However, additional inflow to the 
luminal space occurs in the first side hole of the stent shaft, contrary to case 1 in which the model does not have any side holes in the stent shaft. The streamline of urine flow around the ports and side holes in case 2 is demonstrated in Figure 5.

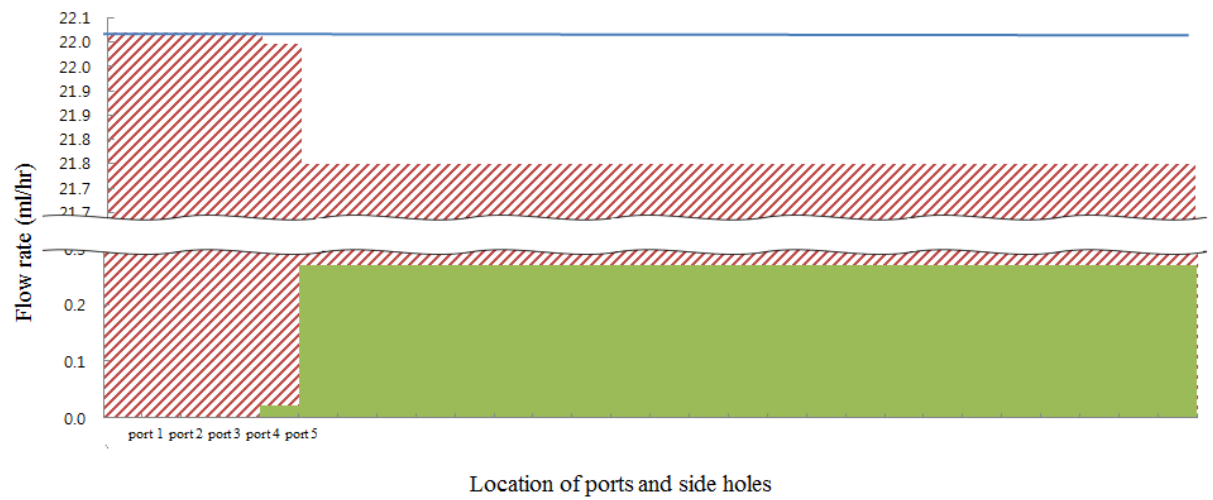

Fig. 3. Individual flow rates in case 1. The upper margins of the green and comb-pattern red areas present the luminal and extraluminal flow rates, and the upper blue line represents total flow rate. There is no side hole in the shaft of the stent. Luminal flow occurs mainly through port 5 and partially through port 4.

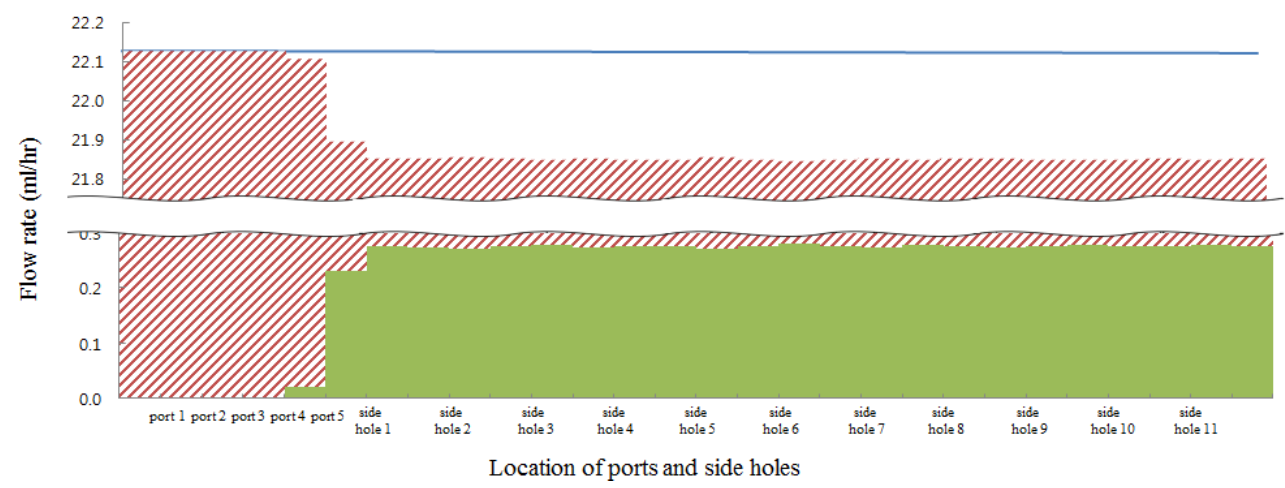

Fig. 4. Individual flow rates in case 2. The upper margins of the green and comb-pattern red areas present the luminal and extraluminal flow rates, and the upper blue line represents total flow rate. Urine also flows into the stent through side hole 1.

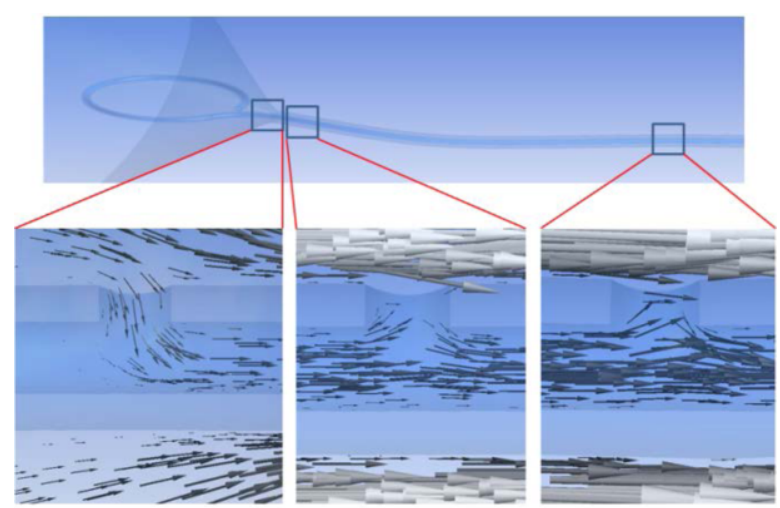

Fig. 5. Flow pattern around ports and side holes of the stent in case 2. Urine flows into the stent through the fifth port and the first side hole is shown in magnified views, but no flow into or out of the stent exists through the fifth side hole. 


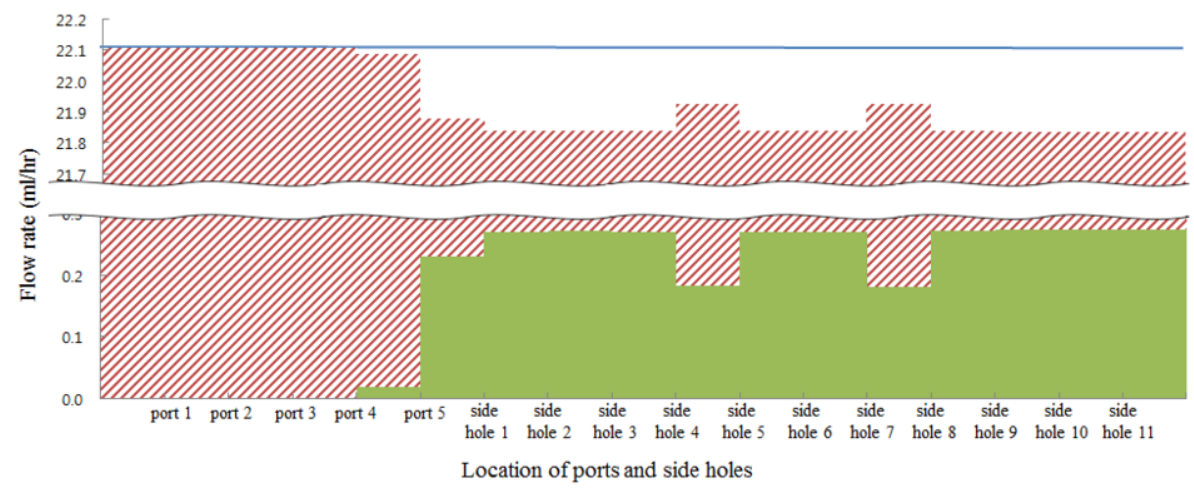

Fig. 6. Individual flow rates in case 6. The upper margins of the green and comb-pattern red areas present luminal and extraluminal flow rates, and the upper blue line represents total flow rate. A decrease and increase in the luminal flow rate are shown before and after each of the two in-stent stenoses between side holes 4 and 5 and between side holes 7 and 8 .

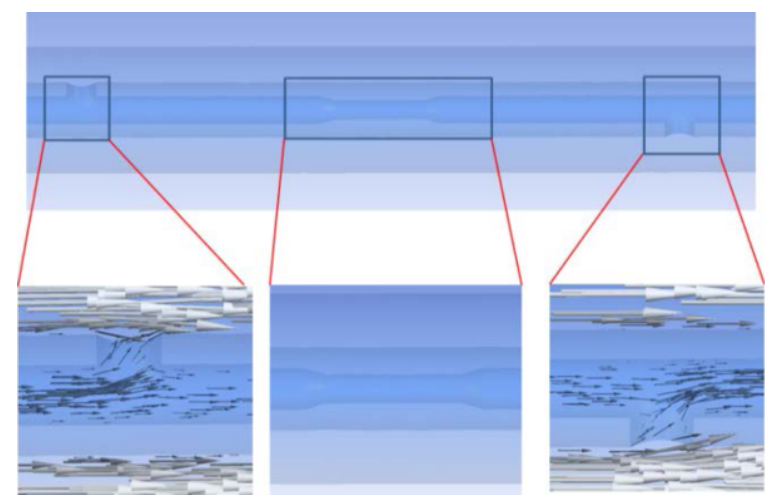

Fig. 7. Flow pattern at side holes around in-stent stenosis in case 6 . Urine flow out of the stent through the side hole before stenosis is shown in magnified views and flow into the stent through the side hole after stenosis is also demonstrated.

In the evaluation of individual flow rates, the gap between the luminal and extraluminal flow rates was very large and the extraluminal flow rate was at least 60 times higher than the luminal flow rate. The big difference originated from the larger diameter of the ureter compared to the inner diameter of the stent. The ratio of the dimension of the luminal and extraluminal space was $(4.57 \times 4.57-2 \times 2)$ $\mathrm{mm}^{2}$-to-1 $\times 1 \mathrm{~mm}^{2}$. It seems realistic, because the models used in the study were made based on the real size of the ureter in a human body. The large ureteral diameter affected the flow rates in the inner and outer space of the stent and most urine flowed in the outer space. For this reason, in a case of notso-tight ureteral stenosis (case 5), the role of a ureteral stent is very limited.

The pattern of the individual flow rates in cases 3 and 4 were similar to that in case 2, although they have a different number and position of side holes in the stent shaft. This means the change in the number and position of side holes did not make any significant difference in the urine flow through the stented ureter. Cases 5 and 6 , as mentioned in the methods, simulated the stenosis in the ureter by a tumorous condition at or around the ureter and in the stent by luminal encrustation. The ureteral stenosis in case 5 did not affect the luminal and extraluminal flow rates and the pattern of the individual flow rates was similar to that in case 2 . The severity of the ureteral stenosis simulated here was not high enough to make a detouring flow from the extraluminal to the luminal space. Further 
study of the effects of different severities of ureteral stenosis on flow rate and pattern is needed in the future.

According to the results of this study, a ureteral stent has a limited role in a view of the size of the inner space of a stent and the flow rate through the space. For example, the use of a stent for total ureteral occlusion could be meaningless because luminal flow is too small to relieve the stagnation of urine in the renal pelvis, and stents differing from plastic stents such as double $\mathrm{J}$ stents used here, might need to be applied for total or tight ureteral stenosis. However, the severity of ureteral stenosis used in this study could be different from real clinical settings and a double $\mathrm{J}$ stent could show better results in the settings. This presents another reason to conduct further study on the effects of different severities of ureteral stenosis on flow rate and pattern.

It might be better to consider again the role of ureteral stents for ureteral stenosis or occlusion. Maintaining urine flow with a stent seems to depend mainly on extraluminal flow supported by a double $\mathrm{J}$ stent, preventing total ureteral occlusion [5, 13]. If the stent could not maintain the extraluminal space, the role of the stent in urine flow rate becomes too small, because the luminal flow out of the total flow in the stented ureter is very small. In the setting, other stents or other treatment methods should be considered to relieve the insufficient urine flow from the kidney to the bladder.

Contrary to cases $1-4$, which did not show any role of the multiple side holes, except the proximal first hole, case 6 showed the role of multiple side holes. The luminal stenosis simulating encrustation caused a bypass of urine flow from the inner space to the outer space of the stent through the side hole proximal to the stenosis, as well as the restoration of urine flow from the outer space to the inner space through the side hole distal to the stenosis (Figures 6 and 7).

\section{Conclusions}

This study could be considered unique in the numerical analysis of urine flow in the stented ureter in that it was performed using a model based on the human anatomy, as well as in that the results expected from the model could be close to real data achieved from a human body. In this study, the total flow rates in the curved and straight models were different, suggesting curved models based on the human anatomy are more desirable in CFD simulations of urine flow. The study also suggests multiple side holes in a stent shaft are needed to relieve the stent stenosis effectively. However, the exact number and precise positions of the side holes should be studied further.

The limitation in the role of double $\mathrm{J}$ stents was shown in this study. To overcome this limitation, the increase in the inner diameter of a stent could be suggested, because just a 1-mm inner diameter of a stent supplies a very small luminal flow rate and the flow rate is not enough to generate sufficient urine flow from the kidney to the bladder [14]. Although it could be a good idea to generate sufficient urine flow through the stent, the insertion of a stent with a larger diameter through nephrostomy or endoscopy could be troublesome.

As seen in this study, CFD simulation serves as a powerful tool to evaluate the effect of geometrical changes in stent design on urine flow and to generate new information and apply it to creating a new stent. In addition, it enables a more rational and reasonable approach to stent design. The models set up here must be applicable for use in evaluating the effects of geometrical variations in stent design on urine flow. 


\section{Acknowledgment}

This research was supported by the Basic Science Research Program through the National Research Foundation of Korea (NRF) funded by the Ministry of Science, ICT \& Future Planning (2014R1A1A1003987).

\section{References}

[1] B. Vahidi and N. Fatouraee, A numerical simulation of peristaltic motion in the ureter using fluid structure interactions, Proceedings of the $29^{\text {th }}$ Annual International Conference of the IEEE EMBS, Cite Internationale, Lyon, France, 2007, pp. $1168-1171$.

[2] H.H. Chung, K.D. Kim, J.Y. Won, J.H. Won, S.B. Cho, T.S. Seo, S.W. Park and B.C. Kang, Multicenter experience of the newly designed covered metallic ureteral stent for malignant ureteral occlusion: comparison with double $\mathrm{J}$ stent insertion, Cardiovascular Interventional Radiology 37 (2014), 463-470.

[3] A. Al-Aown, I. Kyriazis, P. Kallidonis, P. Kraniotis, C. Rigopoulos, D. Karnabatidis, T. Petsas and E. Liatsikos, Ureteral stents: new ideas, new designs, Therapeutic Advances in Urology 2 (2010), 85-92.

[4] N. Venkatesan, S. Shroff, K. Jayachandran and M. Doble, Polymers as ureteral stents, Journal of Endourology 24 (2010), 191-198.

[5] J.W. Ramsay, S.R. Payne, P.T. Gosling, H.N. Whitfield, J.E. Wickham and D.A. Levison, The effects of double J stenting on unobstructed ureters: An experimental and clinical study, British Journal of Urology 57 (1985), 630-634.

[6] A.C. Kinn and H. Lykkeskov-Andersen, Impact on ureteral peristalsis in a stented ureter: An experimental study in the pig, Urological Research 30 (2002), 213-218.

[7] U. Patel and M.J. Kellett, Ureteric drainage and peristalsis after stenting studied using colour doppler ultrasound, British Journal of Urology 77 (1996), 530-535.

[8] J.C. Tong, E.M. Sparrow and J.P. Abraham, Numerical simulation of the urine flow in a stented ureter, Journal of Biomechanical Engineering 129 (2007), 187-192.

[9] J.H. Siggers, S. Waters, J. Wattis and L. Cummings, Flow dynamics in a stented ureter, Mathematical Medicine and Biology 26 (2009), 1-24.

[10] L.J. Cummings, S.L. Waters, J.A. Wattis and S.J. Graham, The effect of ureteric stents on urine flow: Reflux, Journal of Mathematical Biology 49 (2004), 56-82.

[11] F. Clavica, X. Zhao, M. ElMahdy, M.J. Drake, X. Zhang and D. Carugo, Investigating the flow dynamics in the obstructed and stented ureter by means of a biomimetic artificial model, PLoS One 9 (2014), e87433.

[12] D. Carugo, X. Zhang, J.M. Drake and F. Clavica, Formation and characteristics of laminar vortices in microscale environments within an obstructed and stented ureter: a computational study, $18^{\text {th }}$ International Conference on Miniaturized Systems for Chemistry and Life Sciences, MicroTAS, San Antonio, USA, 2014, pp. 1056-1058.

[13] J.W. Ramsay and H.N. Whitfield, Bilateral pelviureteric junction obstruction: the case for endoscopic management, Journal of the Royal Society of Medicine 78 (1985), 590-593.

[14] A.V. Brewer, A.M. Elbahnasy, E. Bercowsky, K.L. Maxwell, A.L. Shalhav, S.A. Kahn, E.M. McDougall and R.V. Clayman, Mechanism of ureteral stent flow: A comparative in vivo study, Journal of Endourology 13 (1999), $269-271$. 